ues of matrices seems to be a paper by Carmichael [3] in 1921. Other examples include $[\mathbf{1}, \mathbf{2}, \mathbf{6}, \mathbf{7}]$. Such work typically considers systems of the form

$$
\sum_{j=1}^{k} \lambda_{j} A_{i j} \mathbf{x}_{i}=\mathbf{0},
$$

where each $A_{i j}$ is an $m_{i} \times n_{i}$ matrix and $\mathbf{x}_{i}$ is an $n_{i}$-element vector. We seek $k$-tuples $\left(\lambda_{j}\right)_{j=1}^{k}$ such that (8) can be solved with each $\mathbf{x}_{i}$ nonzero. Much of this work is at quite a general level and there seems to be little explicit discussion of the interesting special case considered here. We have focused on the properties of this special case, which we believe deserves to be better known.

Acknowledgment. We thank the referees for their helpful comments.

\title{
REFERENCES
}

1. F. V. Atkinson, Multiparameter spectral theory, Bull. Amer. Math. Soc. 74 (1968), 1-27.

2. P. Binding and P. J. Browne, Two parameter eigenvalue problems for matrices, Linear Algebra Appl. 113 (1989), 139-157.

3. R. D. Carmichael, Boundary value and expansion problems: algebraic basis of the theory, Amer. J. Math. 43 (1921), 69-101.

4. T. L. Heath, The Thirteen Books of Euclid's Elements, Vol. 2: Books III-IX (2nd edn.), Dover, New York, 1956.

5. V. V. Prasolov, Problems and Theorems in Linear Algebra, Translations of Mathematical Monographs 134, Amer. Math. Soc., Providence, RI, 1994.

6. B. D. Sleeman, Multiparameter Spectral Theory in Hilbert Space, Research Notes in Mathematics 22, Pitman, London, 1978.

7. H. Volkmer, Multiparameter Eigenvalue Problems and Expansion Theorems, Lecture Notes in Mathematics 1356, Springer-Verlag, Berlin, 1988.

\section{The Volume Swept Out by a Moving Planar Region}

\author{
ROBERT L. FOOTE \\ Wabash College \\ Crawfordsville, IN 47933 \\ footer@wabash.edu
}

I would like to call attention to a beautiful theorem about volume. The only place I have found it is in Courant's calculus text [3, p. 295], [4, p. 451]. Given that this book is a classic and that the result is both simple and elegant, it is surprising that it has not appeared in every calculus text since.

The result generalizes both Cavalieri's Principle and the Theorem of Pappus as a means for computing the volume swept out by a moving planar region. Somewhat informally, let $S_{t}, a \leq t \leq b$, be a planar region of area $A(t)$ moving in space. Let $\mathbf{n}(t)$ be a continuous unit vector normal to the plane of the region, and let $\mathbf{v}(t)$ be the velocity of the centroid of $S_{t}$. Then the signed (or oriented) volume swept out by $S_{t}$ is

$$
V=\int_{a}^{b} A(t) \mathbf{n}(t) \cdot \mathbf{v}(t) d t .
$$

Intuitively, the volume is signed in the following sense. The vector $\mathbf{n}(t)$ indicates an orientation or forward direction (see FIGURE 1). Volume swept out in this direction is 


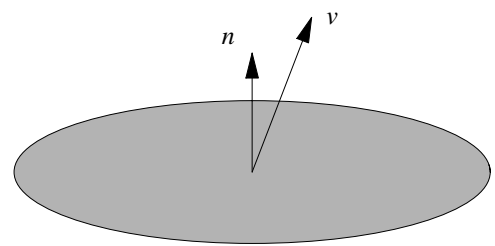

Figure 1 The velocity $\mathbf{v}$ and forward direction $\mathbf{n}$ of a moving planar region

taken to be positive; volume swept out in the opposite (or backward) direction is taken to be negative. This is handled in the integrand of (1) by the sign of $\mathbf{n} \cdot \mathbf{v}$. The net signed volume swept out is, in general, the result of both forward and backward motions. The formula also allows for multiplicities: a point in the path of the moving region may be covered more than once and in both forward and backward directions, in which case the volume of some neighborhood of the point is counted in (1) accordingly.

A recent article by England and Miller [5] gives a variation of (1) that is both more general, in that it allows the reference curve $\boldsymbol{\gamma}$ to be one other than the path of the centroid, and more specialized, in that it requires $\gamma^{\prime}(t)$ to be perpendicular to the plane of $S_{t}$. The result is a nice formula for the signed volume swept out in terms of the geometry of $\gamma$ and its relationship to the centroid of $S_{t}$. A proof of their result is given below (Theorem 2) as an application of (1).

The familiar Theorem of Pappus and Cavalieri's Principle are easily seen to be special cases of (1).

Theorem of PAppus. Suppose $S$ is a bounded planar region of area A that is revolved about a line $\ell$ lying in the plane of $S$. If $S$ lies in one of the half-planes bounded by $\ell$, then the volume of the solid of revolution swept out by $S$ is $2 \pi r A$, where $r$ is the radius of the circle swept out by the centroid of $S$.

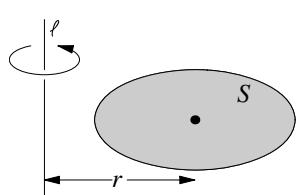

(a)

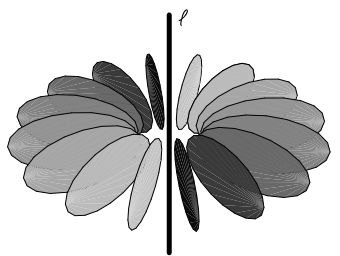

(b)

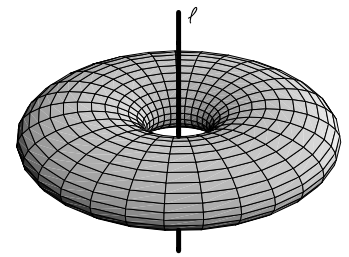

(c)

Figure 2 Theorem of Pappus

FIgURE 2a shows a region $S$ and its centroid. FIgURE 2 b shows $S$ rotating about a line $\ell$, resulting in the solid in FIGURE 2c. Since the area is constant and $\mathbf{n}$ can be taken to be $\mathbf{v} /\|\mathbf{v}\|$, the integral in (1) reduces to $A$ times the distance moved by the centroid. Since every point of $S$ moves in the forward direction, the signed volume is all positive.

The volume formula in the Theorem of Pappus is valid, in fact, even when $\ell$ passes through $S$, as in FIGURE 3a, as long as it is interpreted as signed volume. The line $\ell$ divides $S$ along a chord into two subregions. As $S$ rotates about this chord, the two subregions move in opposite directions, one moving forward and the other backwards. Each subregion generates a solid, shown in FIGURES $3 \mathrm{c}$ and $3 \mathrm{~d}$. Then (1) implies that $2 \pi r A$ is the difference of the volumes of these two solids. This illustrates that a 


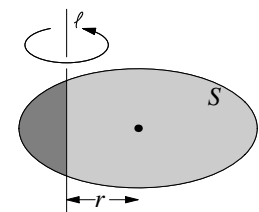

(a)

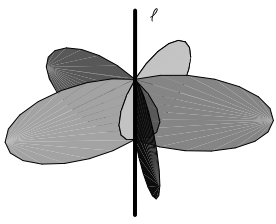

(b)

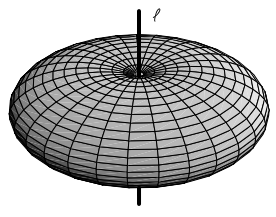

(c)

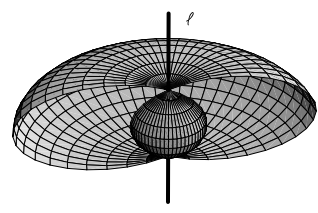

(d)

Figure 3 Generalized Theorem of Pappus

moving region can simultaneously generate both positive and negative signed volumes, an observation that is key to interpreting the integrand of (1).

Cavalieri's Principle states that if two solids have equal cross-sectional areas when cut by any plane parallel to a given plane, then the solids have the same volume (FIGURE 4). This is true even if the cross sections of one are stacked up straight and those of the other are skewed. What is important is not the exact path of the crosssectional centroid, but rather the component of its motion perpendicular to the family of planes, which is computed by the dot product in (1). If the Theorem of Pappus is about rotations, Cavalieri's Principle, in contrast, is about translations. Since the cross sections are parallel, two nearby cross sections are (approximately) translates of each other.
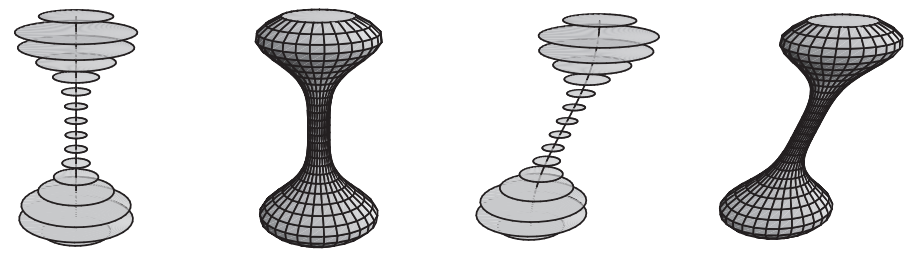

Figure 4 Cavalieri's Principle

Courant states (1) without proof. Instead, he states and proves its analog for the signed area swept out by a line segment moving in $\mathbb{R}^{2}$,

$$
A=\int_{a}^{b} L(t) \mathbf{n}(t) \cdot \mathbf{v}(t) d t
$$

where $L$ is the length of the segment, $\mathbf{n}$ is a forward-pointing unit normal vector, and $\mathbf{v}$ is the velocity of the midpoint, as in FIGURE 5. He goes on to use this to explain how a planimeter works (for more about planimeters, see $[6,7,8]$ ).

One can think of the integrand of (1) as the infinitesimal signed volume swept out due to an infinitesimal motion of the region (with a similar interpretation for the inte-

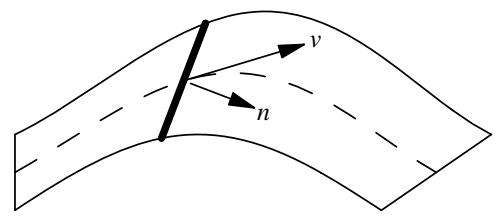

Figure 5 Moving segment sweeping out area in $\mathbb{R}^{2}$ 
grand of (2)). The simplicity of this expression hides the fact that even an infinitesimal motion can result in a combination of both positive and negative volumes. This happens, for example, for the region $S$ in FIgURE 3 ab as it rotates about the line $\ell$. In general it happens when the region $S_{t}$ rotates slightly to $S_{t+d t}$ about one of its chords, $\ell_{t}$, as in FIGURE 6. (For the purpose of this informal discussion we take a chord of $S_{t}$ to be a line in the plane of $S_{t}$ such that there are points of $S_{t}$ on both sides of the line. This agrees with the usual notion of chord when the region is connected.) Part of the content of (1) and its proof is that only the displacement $\mathbf{v} d t=d \mathbf{x}=\mathbf{T} d s$ of the centroid relative to the direction of the normal $\mathbf{n}$ matters.

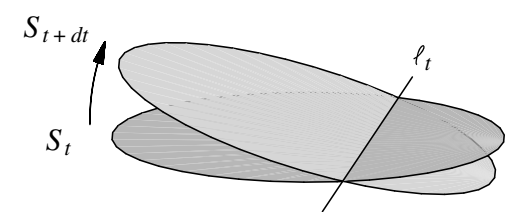

Figure 6 Infinitesimal rotation about a chord

I find the result in $\mathbb{R}^{2}$ less intriguing - the formula is almost obvious due to the symmetry of a line segment about its midpoint. The "symmetry" of a planar region about its centroid is more subtle. For example, a chord through the centroid generally doesn't bisect the area of the region. (A chord through the centroid of a triangle parallel to one of the sides divides the area in the ratio 4:5.) It follows from (1), however, that if the region rotates about such a chord, the signed volume swept out is zero. Consequently, (1) gives some insight into the geometric significance of the centroid that complements the physical center-of-mass interpretation in many calculus texts.

Definitions and proof To prove (1), we first make the notion of signed volume more precise. Suppose $U \subset \mathbb{R}^{3}$ is a bounded region, $F: U \rightarrow \mathbb{R}^{3}$ is $C^{1}$, and $F(U)$ is bounded. We take the signed volume covered by $F$ to be the value of $\iiint_{U} J_{F} d V$, where $J_{F}=\operatorname{det} D F$ is the Jacobian determinant. It's clear that if $F$ is one-to-one on $U^{+}=\left\{\mathbf{x} \in U: J_{F}(\mathbf{x})>0\right\}$ and on $U^{-}=\left\{\mathbf{x} \in U: J_{F}(\mathbf{x})<0\right\}$, then the signed volume covered by $F$ is the volume of $F\left(U^{+}\right)$minus the volume of $F\left(U^{-}\right)$. (By Sard's Theorem [11], the image of $U^{0}=\left\{\mathbf{x} \in U: J_{F}(\mathbf{x})=0\right\}$ has volume 0 even if the volume of $U^{0}$ is positive.) If $F$ is finite-to-one on $U^{+} \cup U^{-}$, the signed volume takes into account the multiplicity of the coverings. The signed volume swept out by the moving planar region in Theorem 1 is to be taken in this sense, as will be clear in the proof.

THEOREM 1. Let $P_{t}, a \leq t \leq b$, be a family of planes. For each $t$ suppose $S_{t}$ is a region in $P_{t}$ such that $S_{t}$ varies continuously with $t$. Let $A(t)$ be the area of $S_{t}$, let $\mathbf{c}(t)$ be the centroid of $S_{t}$, and let $\mathbf{n}(t)$ be a unit normal to $P_{t}$. Assume $\mathbf{c}$ and $\mathbf{n}$ are $C^{1}$, and that $\cup_{t} S_{t}$ is bounded. Then the signed volume swept out by $S_{t}$ is given by (1) (repeated here for emphasis), where $\mathbf{v}(t)=\mathbf{c}^{\prime}(t)$.

$$
V=\int_{a}^{b} A(t) \mathbf{n}(t) \cdot \mathbf{v}(t) d t .
$$

The proof is similar to that given by England \& Miller [5], but somewhat simpler. Note that much of the notation introduced in the proof is used in the remainder of the paper.

Proof. Let $\mathbf{e}_{1}(t), \mathbf{e}_{2}(t)$, and $\mathbf{e}_{3}(t)$ form a $C^{1}$, positively-oriented, orthonormal frame along $\mathbf{c}$ with $\mathbf{e}_{1}(t)=\mathbf{n}(t)$. (To get this one could, for example, apply the Gram-Schmidt 
process to $\mathbf{n}(t)$ and $\mathbf{i}$ to obtain $\mathbf{e}_{1}(t)$ and $\mathbf{e}_{2}(t)$, assuming $\mathbf{n}(t) \neq \mathbf{i}$ for all $t$, and then let $\mathbf{e}_{3}(t)=\mathbf{e}_{1}(t) \times \mathbf{e}_{2}(t)$.)

Define $F:[a, b] \times \mathbb{R}^{2} \rightarrow \mathbb{R}^{3}$ by

$$
F(t, x, y)=\mathbf{c}(t)+x \mathbf{e}_{2}(t)+y \mathbf{e}_{3}(t) .
$$

Note that $F(t, 0,0)=\mathbf{c}(t)$, that is, $F$ maps the $t$-axis to the path of the centroid. For $t=t_{0}$ fixed, note that $\left\{\left(t_{0}, x, y\right):(x, y) \in \mathbb{R}^{2}\right\}$ is the plane in $\mathbb{R}^{3}=\mathbb{R} \times \mathbb{R}^{2}$ perpendicular to the $t$-axis at $t_{0}$. Similarly, $\left\{F\left(t_{0}, x, y\right):(x, y) \in \mathbb{R}^{2}\right\}$ is the plane in $\mathbb{R}^{3}$ passing through $\mathbf{c}\left(t_{0}\right)$ perpendicular to $\mathbf{n}\left(t_{0}\right)$, which is the plane $P_{t_{0}}$ containing $S_{t_{0}}$.

For each $t$, let $\tilde{S}_{t}=\left\{(t, x, y): F(t, x, y) \in S_{t}\right\}$. Since $\mathbf{e}_{2}(t)$ and $\mathbf{e}_{3}(t)$ are orthonormal, then $\tilde{S}_{t}$ and $S_{t}$ are congruent. Furthermore, the centroid of $\tilde{S}_{t}$ is $(t, 0,0)$. Thus the map $F$ achieves a straightening out of the moving region $S_{t}$ into the moving region $\tilde{S}_{t}$ that stays perpendicular to a fixed direction (the $t$-axis), and so that the centroid of $\tilde{S}_{t}$ moves in a straight line with constant unit speed. The moving region $\tilde{S}_{t}$ sweeps out a bounded region $\tilde{\Omega}$ in $\mathbb{R} \times \mathbb{R}^{2}$.

The signed volume swept out by $S_{t}$, that is, the signed volume covered by $\left.F\right|_{\tilde{\Omega}}$, is

$$
V=\iiint_{\tilde{\Omega}} J_{F} d t d x d y=\iiint_{\tilde{\Omega}} \frac{\partial F}{\partial t} \cdot\left(\frac{\partial F}{\partial x} \times \frac{\partial F}{\partial y}\right) d t d x d y .
$$

We have

$$
\frac{\partial F}{\partial t}=\mathbf{v}(t)+x \mathbf{e}_{2}^{\prime}(t)+y \mathbf{e}_{3}^{\prime}(t), \quad \frac{\partial F}{\partial x}=\mathbf{e}_{2}(t), \quad \text { and } \quad \frac{\partial F}{\partial y}=\mathbf{e}_{3}(t) .
$$

Using $\mathbf{e}_{2}(t) \times \mathbf{e}_{3}(t)=\mathbf{e}_{1}(t)=\mathbf{n}(t)$, we have

$$
J_{F}=\frac{\partial F}{\partial t} \cdot\left(\frac{\partial F}{\partial x} \times \frac{\partial F}{\partial y}\right)=\mathbf{v}(t) \cdot \mathbf{n}(t)+x \mathbf{e}_{2}^{\prime}(t) \cdot \mathbf{n}(t)+y \mathbf{e}_{3}^{\prime}(t) \cdot \mathbf{n}(t) .
$$

Integrating over $\tilde{\Omega}$, we get

$$
\begin{aligned}
V=\iiint_{\tilde{\Omega}} J_{F} d t d x d y=\int_{a}^{b}\left[\iint_{\tilde{S}_{t}} J_{F} d x d y\right] d t \\
=\int_{a}^{b}\left[\mathbf{v}(t) \cdot \mathbf{n}(t) \iint_{\tilde{S}_{t}} d x d y\right. \\
\left.\quad \quad+\mathbf{e}_{2}^{\prime}(t) \cdot \mathbf{n}(t) \iint_{\tilde{S}_{t}} x d x d y+\mathbf{e}_{3}^{\prime}(t) \cdot \mathbf{n}(t) \iint_{\tilde{S}_{t}} y d x d y\right] d t .
\end{aligned}
$$

Now, $\iint_{\tilde{S}_{t}} d x d y$ is the area of $\tilde{S}_{t}$, which is $A(t)$. Furthermore, $\iint_{\tilde{S}_{t}} x d x d y$ and $\iint_{\tilde{S}_{t}} y d x d y$ are both zero, since the centroid of $\tilde{S}_{t}$ is $(t, 0,0)$. Thus the integral reduces to $\int_{a}^{b} A(t) \mathbf{v}(t) \cdot \mathbf{n}(t) d t$, which is the desired result.

As an example, consider the undulating torus swept out by a moving disk of varying radius, pictured in FIGURE 7ab. At time $t \in[0,2 \pi]$ the center of the disk is $\mathbf{c}(t)=$ $4 \mathbf{u}(t)$, where $\mathbf{u}(t)=\cos t \mathbf{i}+\sin t \mathbf{j}$. The radius of the disk is $r(t)=1+\frac{1}{2} \cos (3 t)$, and the unit normal to the disk is $\mathbf{n}_{1}(t)=\mathbf{c}^{\prime}(t) / 4$. The volume of the torus is then

$$
V_{1}=\int_{0}^{2 \pi} A(t) \mathbf{v}(t) \cdot \mathbf{n}_{1}(t) d t=\int_{0}^{2 \pi} \pi(2+\cos (3 t))^{2} d t=9 \pi^{2} \approx 88.8 .
$$




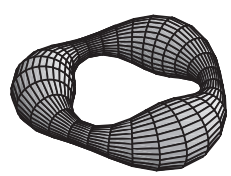

(a)

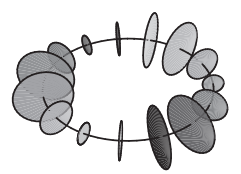

(b)

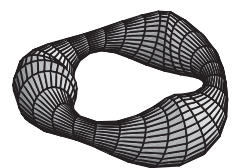

(c)

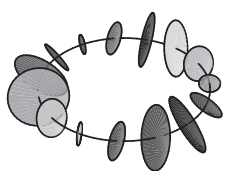

(d)

Figure 7 Moving disks sweeping out volume.

We now perturb the disk so that it wobbles as it sweeps out area, pictured in FIGURE 7cd. Let $\mathbf{N}_{2}(t)=\mathbf{n}_{1}(t)+\frac{1}{2} \sin (3 t) \mathbf{k}+\frac{1}{3} \cos (2 t) \mathbf{u}(t)$ (note that this simply adds to $\mathbf{n}_{1}$ something in its orthogonal complement) and take $\mathbf{n}_{2}(t)=\mathbf{N}_{2}(t) /\left\|\mathbf{N}_{2}(t)\right\|$ as the new unit normal. The new volume is

$$
V_{2}=\int_{0}^{2 \pi} A(t) \mathbf{v}(t) \cdot \mathbf{n}_{2}(t) d t=\int_{0}^{2 \pi} \frac{6 \sqrt{2} \pi(2+\cos (3 t))^{2}}{\sqrt{85+4 \cos (4 t)-9 \cos (6 t)}} d t \approx 82.2,
$$

which is slightly less than the original, as one might expect.

Moving forward One gets volume, as opposed to signed volume, when $J_{F} \geq 0$ on $\tilde{\Omega}$. From the proof, the interpretation of $J_{F}>0$ is that every point of $S_{t}$ moves in the forward direction. In many specific examples this is easy to see by inspection, but it's good to know conditions that imply it. Especially useful conditions are ones that can be applied directly to $S_{t}$, as opposed to $\tilde{S}_{t}$ or $F$.

Note, from (3), that $J_{F}$ is linear in $x$ and $y$. Consequently, if $J_{F}$ is zero at some interior point of $\tilde{S}_{t}$, but not identically zero on $\tilde{S}_{t}$, then it is zero along a whole chord $\tilde{C}$ of $\tilde{S}_{t}$ and takes opposite signs on opposite sides of the chord. This is the infinitesimal version of the fact that if $S_{t_{1}}$ and $S_{t_{2}}$ intersect, they do so along a common chord. To see this, let $C=F(\tilde{C})$ be the corresponding chord of $S_{t}$. Points of $S_{t}$ corresponding to points of $\tilde{S}_{t}$ for which $J_{F}>0$ are moving forward. These points are all on one side of $C$. Points of $S_{t}$ on the opposite side of $C$ are moving backwards. It follows that $C$ is the chord of intersection of $S_{t}$ and $S_{t+d t}$ in FIGURE 6, and $F$ fails to be one-to-one on any neighborhood of any point of $\tilde{C}$. Thus, to conclude that (1) computes volume when the $S_{t}$ are connected, it suffices to assume that $S_{t_{1}}$ and $S_{t_{2}}$ are disjoint when $t_{1} \neq t_{2}$, with the possible exception of $S_{a}$ and $S_{b}$, which might be identical. In the standard use of the Theorem of Pappus this is handled by the assumption that the planar region $S$ lies in one of the half-planes of the line of rotation.

The following proposition gives a precise condition on $S_{t}$ for $J_{F}>0$ on $\tilde{S}_{t}$.

Proposition. $J_{F}>0$ on $\tilde{S}_{t}$ if and only if $(\mathbf{x}-\mathbf{c}(t)) \cdot \mathbf{n}^{\prime}(t)<\mathbf{v}(t) \cdot \mathbf{n}(t)$ for all $\mathbf{x} \in S_{t}$.

Proof. The proof involves rewriting the expression for $J_{F}$ in (3). Since $\mathbf{e}_{2}$ and $\mathbf{e}_{3}$ are perpendicular to $\mathbf{n}$, we have

$$
\begin{aligned}
0 & =\frac{d}{d t}\left(\left(x \mathbf{e}_{2}(t)+y \mathbf{e}_{3}(t)\right) \cdot \mathbf{n}(t)\right) \\
& =x \mathbf{e}_{2}^{\prime}(t) \cdot \mathbf{n}(t)+y \mathbf{e}_{3}^{\prime}(t) \cdot \mathbf{n}(t)+\left(x \mathbf{e}_{2}(t)+y \mathbf{e}_{3}(t)\right) \cdot \mathbf{n}^{\prime}(t) .
\end{aligned}
$$

Now $\mathbf{c}(t), \mathbf{e}_{2}(t)$ and $\mathbf{e}_{3}(t)$ determine a Euclidean coordinate system on $P_{t}$ in which $(x, y)$ are the coordinates of $\mathbf{x}=\mathbf{c}(t)+x \mathbf{e}_{2}(t)+y \mathbf{e}_{3}(t)$. By substitution into (3), $J_{F}$ 
can be written as

$$
J_{F}=\mathbf{v}(t) \cdot \mathbf{n}(t)-\left(x \mathbf{e}_{2}(t)+y \mathbf{e}_{3}(t)\right) \cdot \mathbf{n}^{\prime}(t)=\mathbf{v}(t) \cdot \mathbf{n}(t)-(\mathbf{x}-\mathbf{c}(t)) \cdot \mathbf{n}^{\prime}(t)
$$

for $\mathbf{x} \in S_{t}$, from which the proposition follows.

A few comments will reveal the geometric significance of the proposition. The inequality is a linear condition on points $\mathbf{x}$ in the plane $P_{t}$ of $S_{t}$. If it holds for all $\mathbf{x} \in S_{t}$, then it holds when $\mathbf{x}$ is the centroid $\mathbf{c}(t)$. In this case we get $\mathbf{v}(t) \cdot \mathbf{n}(t)>0$, which simply says that the centroid must be moving forward.

The cases when $\mathbf{n}^{\prime}(t)=\mathbf{0}$ and $\mathbf{n}^{\prime}(t) \neq \mathbf{0}$ are infinitesimal versions of the hypotheses of Cavalieri's Principle and the Theorem of Pappus. To see this, note that since $\mathbf{n}(t)$ is the unit normal to $P_{t}$, the vector $\mathbf{n}^{\prime}(t)$ is a measure of the rotation of the family of planes. If $\mathbf{n}^{\prime}(t)=0$, then having the centroid move forward is sufficient for every point in $P_{t}$ to do the same. This agrees with intuition-in this case the planes $P_{t}$ and $P_{t+d t}$ are parallel (at least to first order). On the other hand, if $\mathbf{n}^{\prime}(t) \neq 0$, then the planes $P_{t}$ and $P_{t+d t}$ are not parallel, as shown in FIGURE 6. The inequality in the proposition defines a half-plane that contains the centroid. The boundary of the half plane is the line $\ell_{t}$ of intersection of $P_{t}$ and $P_{t+d t}$. (The details of this are not difficult, and are left to the interested reader.) In order for the region $S_{t}$ to be moving forward, it must lie in this half plane.

The interested reader may also verify that the inequality in the proposition is satisfied by the wobbling disk example, and so the signed volume computed is the actual volume of the solid swept out.

A variation The hypotheses used by England and Miller [5] are somewhat different than those in Theorem 1. Instead of following the centroid of the moving region, they follow another reference curve $\boldsymbol{\gamma}$ that is assumed to be perpendicular to the plane of the moving region. Their integral formula for volume involves the geometry of $\boldsymbol{\gamma}$ and the displacement from $\gamma$ to the centroid.

Theorem 2. (England \& Miller [5]) Suppose that $\gamma:[0, \ell] \rightarrow \mathbb{R}^{3}$ is a $C^{2}$ curve parameterized by arc length $s$. Let $P_{s}$ be the plane containing $\gamma(s)$ that is perpendicular to $\gamma^{\prime}(s)$. Let $S_{s}$ be a region in $P_{s}$ that varies continuously with $s$. Let $\mathbf{N}(s)$ be the principal normal vector of $\boldsymbol{\gamma}$ and let $r(s)=(\mathbf{c}(s)-\boldsymbol{\gamma}(s)) \cdot \mathbf{N}(s)$, where $\mathbf{c}(s)$ is the centroid of $S_{s}$. Then the signed volume swept out by $S_{s}$ is

$$
V=\int_{0}^{\ell} A(s)(1-\kappa(s) r(s)) d s .
$$

Note that $r(s)$ is the component of the vector from $\gamma(s)$ to the centroid in the direction of the principal normal.

To prove this we first generalize Theorem 1 and then specialize to the situation of Theorem 2. Under the hypotheses and notation of Theorem 1, suppose that $\gamma$ : $[a, b] \rightarrow \mathbb{R}^{3}$ is a $C^{1}$ curve such that $\gamma(t) \in P_{t}$ for all $t$. Here we do not assume that $\boldsymbol{\gamma}$ is parameterized by arc-length or that $P_{t}$ is perpendicular to $\boldsymbol{\gamma}^{\prime}(t)$. From Theorem 1 the signed volume swept out is

$$
V=\int_{a}^{b} A(t)\left(\boldsymbol{\gamma}^{\prime}(t)+\left(\mathbf{c}^{\prime}(t)-\boldsymbol{\gamma}^{\prime}(t)\right)\right) \cdot \mathbf{n}(t) d t .
$$

Since $\mathbf{c}(t)$ and $\boldsymbol{\gamma}(t)$ are both in $P_{t}$, then $\mathbf{c}(t)-\boldsymbol{\gamma}(t)$ is perpendicular to $\mathbf{n}(t)$ and we have

$$
0=\frac{d}{d t}((\mathbf{c}(t)-\boldsymbol{\gamma}(t)) \cdot \mathbf{n}(t))=\left(\mathbf{c}^{\prime}(t)-\boldsymbol{\gamma}^{\prime}(t)\right) \cdot \mathbf{n}(t)+(\mathbf{c}(t)-\boldsymbol{\gamma}(t)) \cdot \mathbf{n}^{\prime}(t) .
$$


(This is similar to the first step in the proof of the proposition.) Thus

$$
V=\int_{a}^{b} A(t)\left(\boldsymbol{\gamma}^{\prime}(t) \cdot \mathbf{n}(t)+(\boldsymbol{\gamma}(t)-\mathbf{c}(t)) \cdot \mathbf{n}^{\prime}(t)\right) d t .
$$

Now assume the hypotheses of Theorem 2. Since $P_{s}$ is perpendicular to $\boldsymbol{\gamma}^{\prime}(s)$, we may take $\mathbf{n}(s)=\boldsymbol{\gamma}^{\prime}(s)=\mathbf{T}(s)$, the unit tangent vector of $\boldsymbol{\gamma}$. The second factor in the integrand of (4) becomes

$$
\mathbf{T}(s) \cdot \mathbf{T}(s)+(\boldsymbol{\gamma}(s)-\mathbf{c}(s)) \cdot \mathbf{T}^{\prime}(s)=1-(\mathbf{c}(s)-\boldsymbol{\gamma}(s)) \cdot \kappa(s) \mathbf{N}(s)=1-\kappa(s) r(s),
$$

which proves Theorem 2 .

The integrand of (4) has a nice interpretation. It can be written as

$$
d V=A(t) \boldsymbol{\gamma}^{\prime}(t) \cdot \mathbf{n}(t) d t+A(t)(\boldsymbol{\gamma}(t)-\mathbf{c}(t)) \cdot \mathbf{n}^{\prime}(t) d t .
$$

Suppose that the infinitesimal motion of $S_{t}$ is purely translational. Then $\mathbf{n}^{\prime}(t)=\mathbf{0}$ and $\boldsymbol{\gamma}^{\prime}(t) \neq \mathbf{0}$, and the infinitesimal volume swept out is given by the first term of (5). On the other hand, suppose that $S_{t}$ is infinitesimally rotating about some line in $P_{t}$ that passes through $\boldsymbol{\gamma}(t)$. In this case $\mathbf{n}^{\prime}(t) \neq \mathbf{0}$ and $\boldsymbol{\gamma}^{\prime}(t)=\mathbf{0}$, and the infinitesimal volume swept out is given by the second term of (5). Thus (5) is the decomposition of $d V$ into purely translational and rotational parts from the perspective of the reference curve $\gamma$. The distinguishing property of the centroid is that it is the unique choice of $\boldsymbol{\gamma}(t)$ for which only the translational part matters in (5).

Higher dimensions and other geometries Interested readers may enjoy generalizing Theorems 1 and 2 to a moving, codimension-one, flat region sweeping out volume in $\mathbb{R}^{n}$. The generalizations to spherical and hyperbolic geometries are less obvious, however. For a geodesic segment sweeping out area in $S^{2}$ or $H^{2}$, the analog of (2) is

$$
A=\int_{a}^{b} \frac{C(L(t) / 2)}{\pi} \mathbf{n}(t) \cdot \mathbf{v}(t) d t
$$

where $L, \mathbf{n}$, and $\mathbf{v}$ are the same as in (2) and $C(r)$ is the circumference of a circle of intrinsic radius $r$ [6]. For the unit sphere, in which the Gaussian curvature is 1 , we have $C(r)=2 \pi \sin r$, and for the hyperbolic plane with curvature -1 we have $C(r)=$ $2 \pi \sinh r$, but (6) is valid for all constant curvatures. The analogs of (1) and (6) in $S^{n}$ and $H^{n}$ for $n \geq 3$, if they have been worked out, are necessarily more complicated because the notion of centroid (and more generally, center of mass) is less clear in these spaces. The definition and equivalence of the various formulations of centroid and center of mass in $\mathbb{R}^{n}$ depend on the affine structure of $\mathbb{R}^{n}$, which is absent in $S^{n}$ and $H^{n}$. The first notion of center of mass for regions in symmetric spaces was developed by Cartan [2], and has been generalized to other settings (see Berger [1]; Galperin [9] gives an extrinsic definition of center of mass in $S^{n}$ and $H^{n}$ ). The notion is more subtle than in $\mathbb{R}^{n}$ and, in particular, its dynamical properties do not extend (for example, the center of mass of a freely-moving rigid body does not necessarily follow a geodesic), and there are competing notions for a substitute concept in this setting $[10]$.

\section{REFERENCES}

1. M. Berger, Riemannian Geometry During the Second Half of the Twentieth Century, Univ. Lecture Series, Vol. 17, American Mathematical Society, Providence, R.I., 2000. 
2. E. Cartan, Groupes simples clos et ouverts et géométrie riemannienne, J. Math. Pures Appl. 8, (1929), 1-33.

3. R. Courant, Differential and Integral Calculus, II. Originally published in 1934 as Vorlesungen über Differential- und Integralrechnung. Translated by E. J. McShane. Wiley Interscience, New York, 1988.

4. R. Courant and F. John, Introduction to Calculus and Analysis, II, Wiley Interscience, New York, 1974.

5. W. T. England and T. L. Miller, Volumes and cross-sectional areas, this MAGAZINE 74:4 (2001), 288-295.

6. R. L. Foote, Planimeters and isoperimetric inequalities on constant curvature surfaces, preprint.

7. R. L. Foote, Geometry of the Prytz planimeter, Reports on Mathematical Physics 42, no. 1, (1998), $249-271$.

8. R. L. Foote, Planimeters, http://persweb.wabash.edu/facstaff/footer/ Planimeter/Planimeter.htm.

9. G. A. Galperin, A concept of the mass center of a system of material points in the constant curvature spaces, Comm. Math. Phys. 154, (1993), 63-84.

10. A. V. Shchepetilov, Two-body problem on two-point homogeneous spaces, invariant differential operators and the mass center concept, Journal of Geometry and Physics 48, (2003), 245-274.

11. M. Spivak, Calculus on Manifolds, W. A. Benjamin, New York, 1965.

\title{
Equal Sums of Three Fourth Powers or What Ramanujan Could Have Said
}

\author{
RICHARD BLECKSMITH \\ Northern Illinois University \\ DeKalb, IL 60115 \\ richard@math.niu.edu \\ SIMCHA BRUDNO \\ 318 S. Throop St. Apt 204 \\ Chicago, IL 60607
}

\section{Dedicated to our friend John Selfridge}

Math trivia buffs recognize 1729 as the number of the taxi that Hardy took to visit Ramanujan in the hospital. When Hardy complained that it seemed like a typical run of the mill number, Ramanujan countered that 1729 is actually a "very interesting number," because "it is the smallest number expressible as the sum of two cubes in two different ways" $\left(9^{3}+10^{3}\right.$ and $\left.1^{3}+12^{3}\right)$ [5, pp. 12]. Ramanujan could have really surprised his friend by saying something like this: "Besides, Hardy, twice its square is the first number which can be written as the sum of three fourth powers in four different ways."

It turns out that $2 \times 1729^{2}$ is the start of an infinite sequence of numbers with the following remarkable property: If $R(n)$ denotes the number of ways that $n$ can be written as the sum of three fourth powers, then $R(n)$ doubles every time we move on to the next number in the list. Consequently, there exist integers with an arbitrarily large number of representations as sums of three fourth powers.

Our investigation utilizes a simple identity:

$$
x^{4}+y^{4}+(x+y)^{4}=2\left(x^{2}+x y+y^{2}\right)^{2} .
$$

This equation is easily established by straightforward algebraic manipulation. Dickson referred to (1) as "Proth's identity." First published in 1878 [3, p. 657, footnote 227], it is an easy consequence of Candido's identity

$$
\left(x^{2}+y^{2}+(x+y)^{2}\right)^{2}=2\left(x^{4}+y^{4}+(x+y)^{4}\right),
$$

